Article

\title{
Effects of Cold Stress on the Photosynthesis and Antioxidant System of Rhododendron chrysanthum Pall.
}

\author{
Xiaofu Zhou ${ }^{\dagger}$, Silin Chen ', Hui Wu, Hongwei Xu* \\ Jilin Provincial Key Laboratory of Plant Resource Science and Green Production, Jilin Normal University, \\ Siping 136000, China; zhouxiaofu@jlnu.edu.cn (X.Z.); chensl1216@163.com (S.C.); 171610975@qq.com (H.W.) \\ * Correspondence: xuhongwei@jlnu.edu.cn; Tel.: +86-134-0461-1487 \\ + These authors contributed equally to this work
}

\begin{abstract}
Rhododendron chrysanthum Pall., live in Changbai Mountain being exposed to chilling temperature, high light intensities and water scarcity condition. To adapt to the harsh environment, the cold resistance mechanisms of $R$. chrysanthum have been successfully evolved in the long-term adaptive process. In our present work, the methods of proteomics combined with physiological and biochemical analyses were used to investigate the effects of cold stress on the photosynthesis and antioxidant system of Rhododendron chrysanthum Pall. and the molecular mechanisms involved in cold resistance of plants. A total of 153 photosynthesis related proteins were identified in present work, of which 7 proteins including Rubisco large subunit ( $\mathrm{rbcL}$ ) were up-regulated in experiment group (EG) compared with control group (CG). Simultaneously, four chlorophyll fluorescence parameters were measured in present study. The results showed that the maximum photochemical efficiency of photosystem II (Fv/Fm), actual quantum yield of PSII $(\mathrm{Y}(\mathrm{II}))$ and photochemical quenching (qP) were significantly higher in $\mathrm{EG}$, whereas the non-photochemical quenching (NPQ) was notably decreased. Cold stress could lead to a significant reduction in electron transport rate (ETR) accompanied with an increase in excitation pressure (1-qP). The abundance of PetE which involved in the plants photosynthetic electron transfer was also significantly influenced by cold stress. Moreover, the up-regulated expressions and higher levels of enzymatic activities of Glutathione peroxidase (GPX) and Ascorbate peroxidases (APXs) were detected in EG. All these changes which can help plants to survive in low temperature are considered as the crucial parts of cold tolerance mechanisms. These results revealed that photosynthesis and redox adjustment play significant roles in the defense of cold-induced damage.
\end{abstract}

Keywords: Rhododendron chrysanthum Pall.; cold stress; chlorophyll fluorescence; photosynthesis; antioxidant enzymes

\section{Introduction}

As a major stress factor, low temperature has a significant effect on the distribution and production of crops and ornamental plants. It can adversely impact the growth and development of plant by altering the metabolism and physiology processes [1]. Cold temperature will induce a series of related reactions, such as accumulating the ROS, decreasing the photochemical efficiency of photosystem II (PSII) and inhibiting the photosynthesis [2]. Moreover, these processes are coupled with each other and play crucial roles in the metabolism system of plants. Cold resistance capacity of plants is an essential prerequisite for successful living through the winter and is a common but crucial problem encountered in high altitude mountainous areas. Hence the investigation of molecular mechanism response to cold stress in plants is particularly important. 
Chlorophyll fluorescence imaging (CFI) technique is an effective tool for evaluating the sensitive index of plant and has been widely used in many field of abiotic stress [3]. The method of chlorophyll fluorescence measurement is endowed with such advantages as more efficient, nondestructive and flexible, compared with other measurement [4]. Not only can chlorophyll fluorescence reflect the extent of damage in the photo system II (PSII), but also can mirror the intrinsic changes of plants to abiotic stress [5].

Rhododendron chrysanthum Pall. grows above 2,734 m a.s.l. in the Changbai Mountain and lives in a harsh climate with the annual average temperature usually between $-7.3^{\circ} \mathrm{C}$ and $3^{\circ} \mathrm{C}$. In the longterm adaptive evolution, $R$. chrysanthum has evolved cold resistance mechanisms that can adapt it to the tough environment and protect it from frozen injury. Proteomics analyses, chlorophyll fluorescence analyses, antioxidant enzymes activity measurements and western blot analyses were used in our study to investigate the impact of cold stress on photosynthesis and antioxidant system, as well as the mechanism of plants cold stress tolerance.

\section{Results}

\subsection{Effect of cold stress on photosynthesis related protein}

A total of 705 proteins were quantified in present research and the quantitative ratio over 1.3 was considered as up-regulation (UR). 153 proteins related to the process of photosynthesis were identified, of which 63 proteins were mapped to the protein interaction network (Figure 1A). All the UR proteins in the network were shaped as a rhombus. Connectedness and weights of these proteins were distinguished by color. 7 proteins including GAPB, GAPA, LHCA4, LHCB4.1, TLP18.3, TPI and RBCL, were up-regulated in experiment group (EG) after cold treatment.

To ensure and validate reliability of differential proteins obtained from proteomics analysis, the expression of Rubisco large subunit (rbcL) in the leaves of CG and EG after short-term low temperature treatment were detected by Western blot (Figure 1B). The content of rbcL in the EG was significantly higher than that in the CG which was similar with those in proteomics analysis.

A

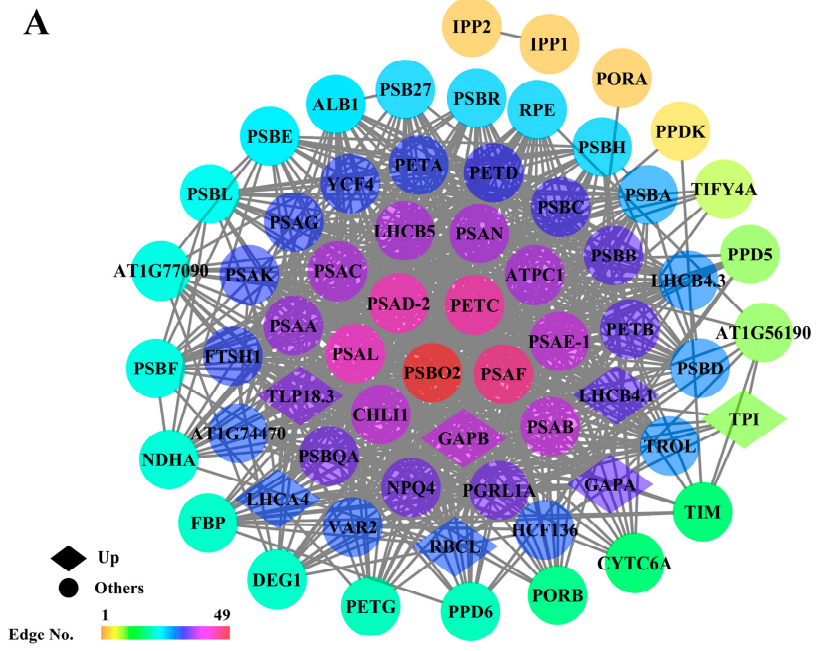

B

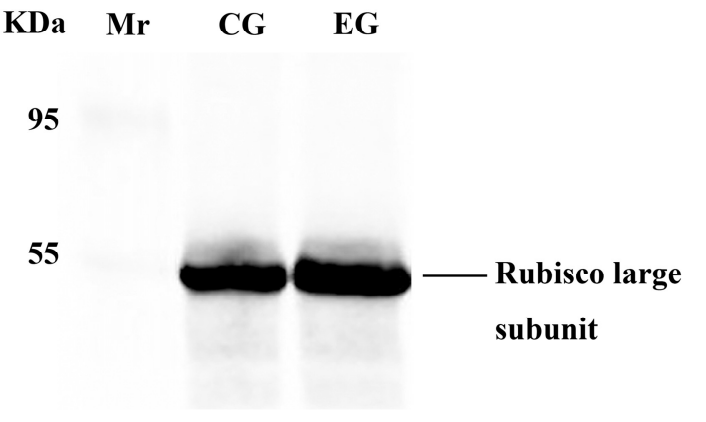

Figure 1. Effect of cold stress on the expression of photosynthesis related protein. (A) Interaction network of photosynthesis related protein in Rhododendron chrysanthum Pall. (B) Western blot analysis of Rubisco large subunit (rbcL). 


\subsection{Effect of cold stress on photochemical activity}

To estimate the effect of cold stress on photosynthesis and explore the relationship between chilling resistance and photosynthetic activities, chlorophyll fluorescence (ChlF) parameters in $R$. chrysanthum were measured using the Imaging-PAM system in present study. Four parameters including the maximum photochemical efficiency of photosystem II (Fv/Fm), actual quantum yield of PSII (Y(II)), non-photochemical quenching (NPQ), and photochemical quenching (qP) were selected for further investigation. The fluorescence images of leaves shown in Figure 2A can indicate the status of the ChlF parameters. In present work, the Fv/Fm, Y(II) and qP of EG were significantly higher after $4^{\circ} \mathrm{C}$ treating for $12 \mathrm{~h}$ than that in CG. However, the value of NPQ was significantly lower in EG (Figure 2B). These results indicated that the cold stress may damage the photosynthetic apparatus and inhibit the photochemical activity of plants. Moreover, the damage degrees were closely associated with the cold tolerance of plants.

A

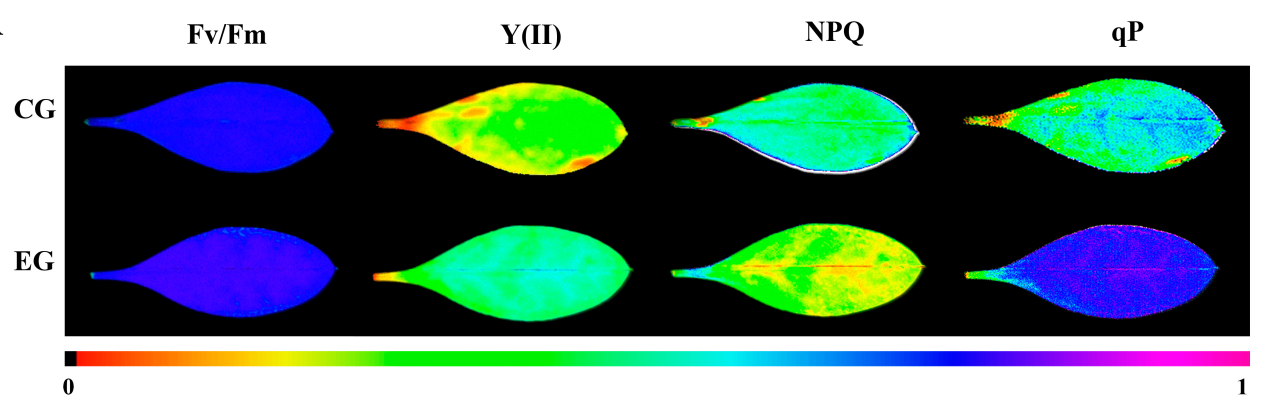

B

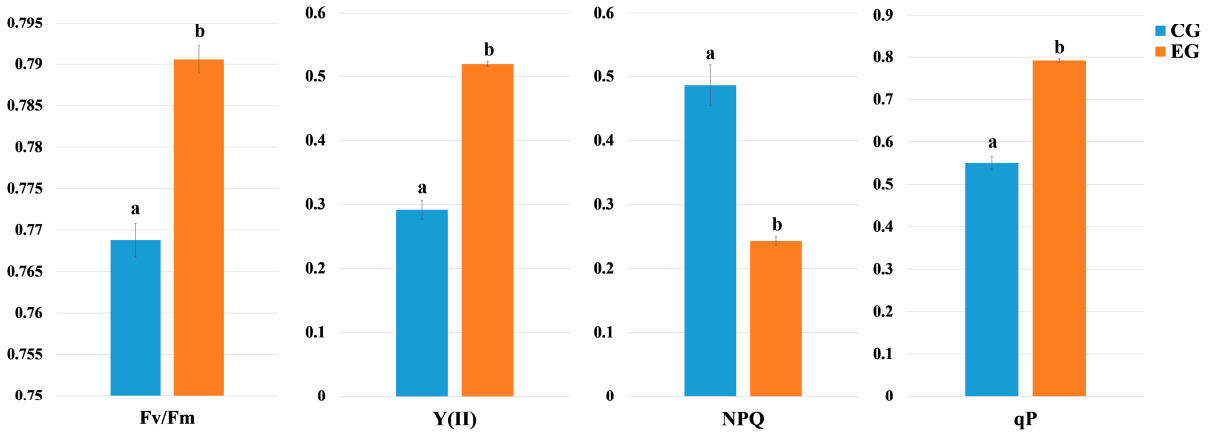

Figure 2. Effect of cold stress on photochemical activity. Chlorophyll fluorescence images (A) and values (B) of maximum quantum efficiency of the photochemistry (Fv/Fm), actual quantum yield of PSII (Y(II)), non-photochemical quenching (NPQ), and photochemical quenching (qP) after $4{ }^{\circ} \mathrm{C}$ treatment for $12 \mathrm{~h}$. The color code in the images ranged from 0 (black) to 1.0 (purple). Values are expressed as means $\pm S D, n=3$. Statistically different values $(p<0.05)$ are indicated by different letters.

\subsection{Effect of cold stress on photosynthetic electron transport}

The rate of electron transport (ETR) in the leaves of EG and CG were measured by using the Imaging-PAM (Heinz Walz, Germany). A significantly higher ETR associated with lower excitation pressure (1-qP) was determined in EG compared with CG (Figure 3A). Moreover, the result of KEGG pathway analysis showed that the PetE ( $P_{c}$, plastocyanin), which plays a significant role in the photosynthesis electron transport of the photosynthesis signaling pathway, was relative upregulated in the EG under cold stress (Figure 3B). These results revealed that the electron transport process of PSII was sensitive to cold stress and cold-tolerance plants can prevent PSII damage by maintaining the stability of electron transfer system under the chilling temperature. 


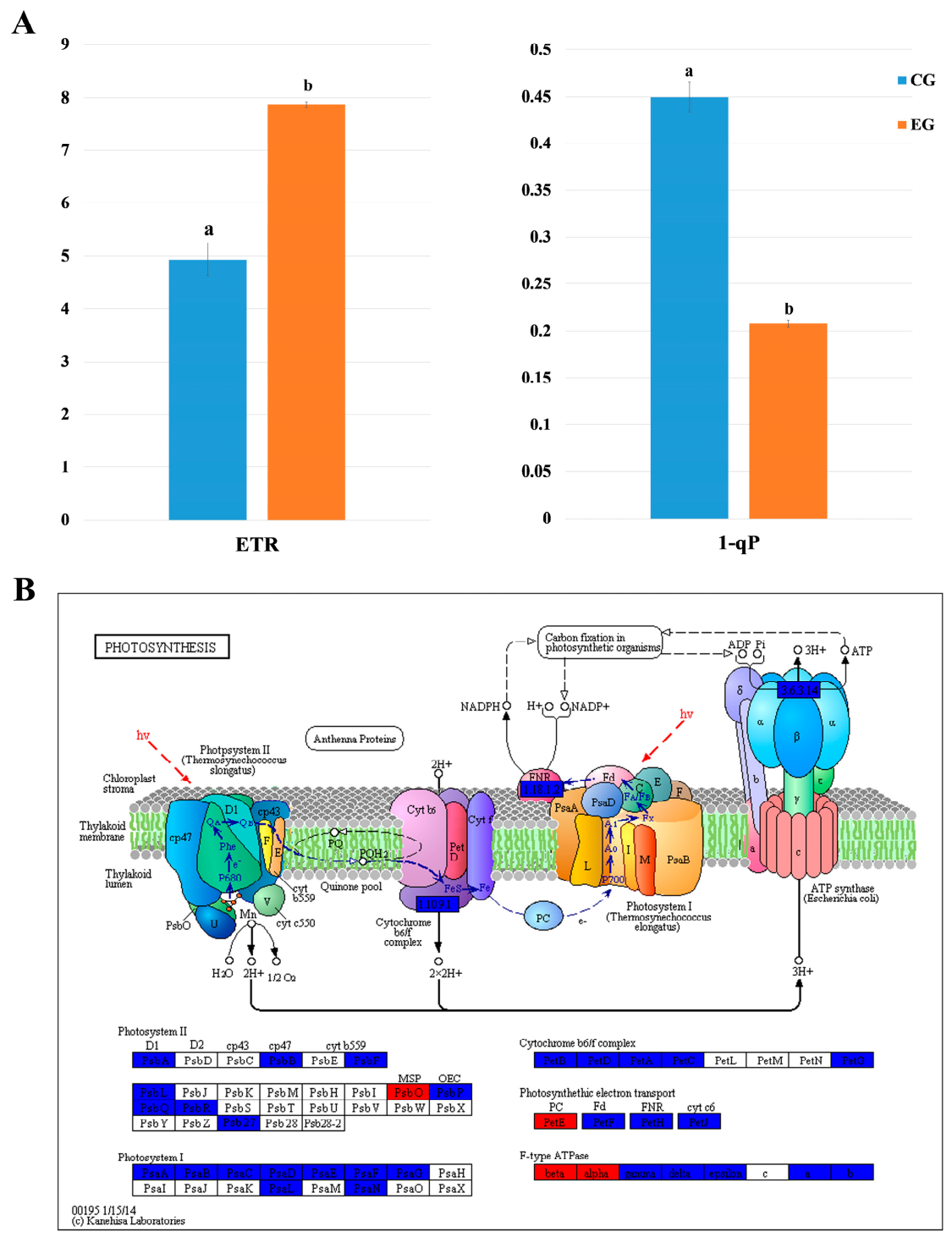

Figure 3. Effect of low temperature treatment on photosynthetic electron transport. (A) Values of electron transport rate (ETR) and the excitation pressure (1- $\mathrm{qP})$. Values are expressed as means $\pm \mathrm{SD}$, $n=3$. Statistically different values $(p<0.05)$ are indicated by different letters. (B) Kyoto encyclopedia of genes and genomes (KEGG) pathway analysis of photosynthesis. Red represents up-regulated proteins in experiment group (ratio $\geq 1.3$ ). Blue represents other quantified proteins.

\subsection{Effect of cold stress on antioxidant system}

To further investigate the molecular mechanism of the plants to defense cold stress, the activities of two antioxidant enzymes Glutathione peroxidase (GPX) and Ascorbate peroxidase (APX) were measured in present work. Meanwhile, the protein interaction network of these mainly antioxidant enzymes and relevant proteins was setting up via Cytoscape software. After $12 \mathrm{~h}$ of $4^{\circ} \mathrm{C}$ treatment, the activities of GPX and APX in EG were 2.6 and 2.3 times higher than that in CG (Figure 4A). Overall, 51 proteins including 5 main proteins (APX1, APX2, SAPX, TAPX, and GPX1) and 46 relevant proteins were mapped in the interaction network (Figure 4B). The connectedness and weights of the proteins in this network were distinguished by color. 11 up-regulated proteins in the networks were shaped as a rhombus and 10 proteins involved in the process of photosynthesis were highlighted by bolder border paint. 
A

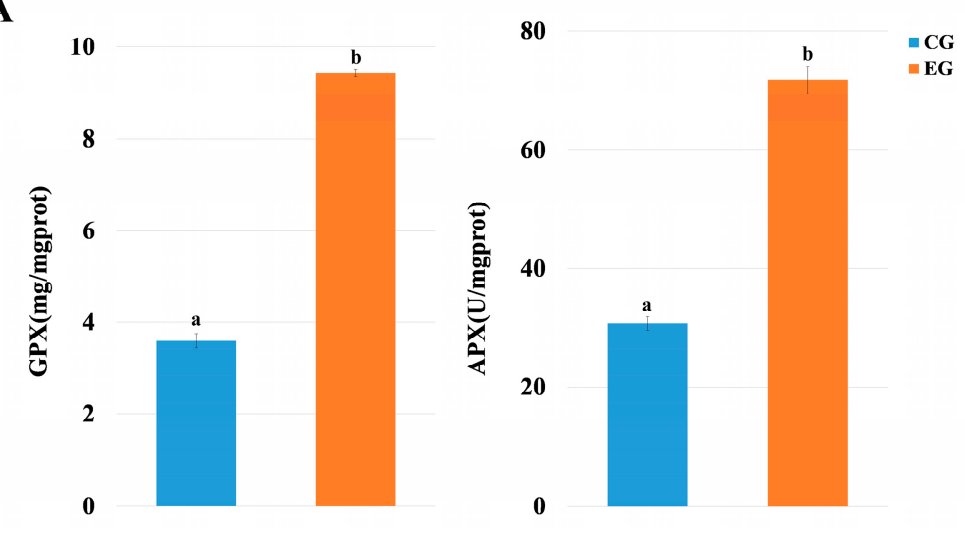

B

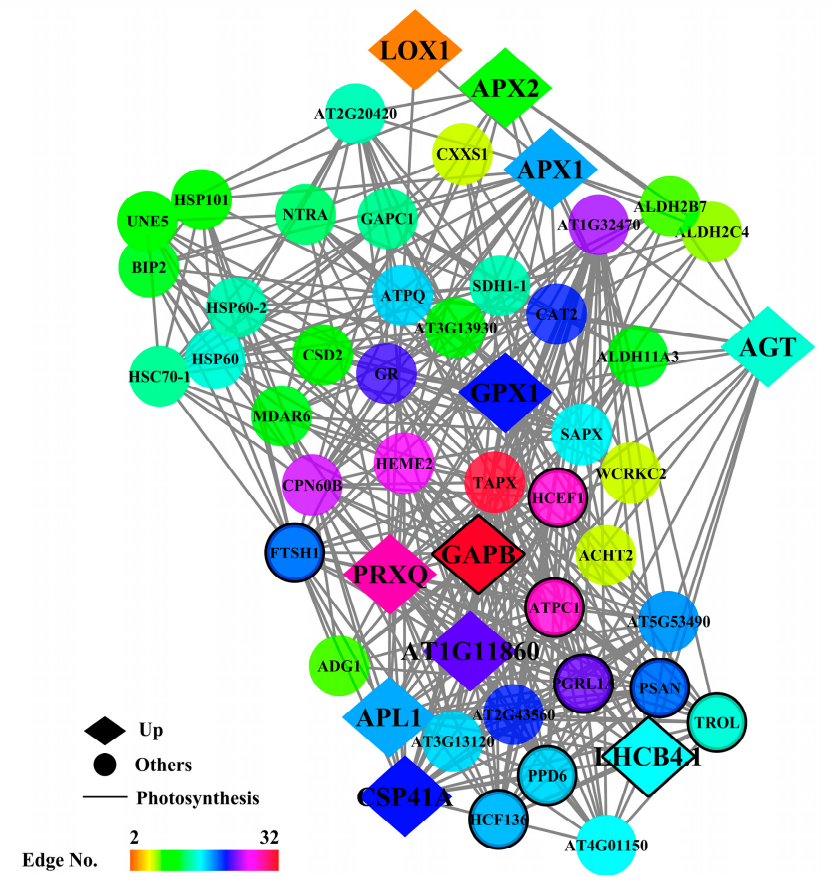

Figure 4. Effect of cold stress on antioxidant system. (A) Activities analyses of glutathione peroxidase (GPX) and ascorbate peroxidases (APX). Values are expressed as means $\pm S D, n=3$. Statistically different values $(p<0.05)$ are indicated by different letters. (B) Protein-protein interaction network of APX, GPX, and their related proteins.

\section{Discussion}

A Cold stress can provoke changes in many aspects of biological process and cellular metabolism, especially in protein synthesis. Low temperature may down-regulate many photosynthesis-related proteins. However, plants with highly resistance to low temperature can adapt the environment by increasing the expression of these proteins. In our study, the expressions of 7 photosynthesis related proteins (GAPB, GAPA, LHCA4, LHCB4.1, TLP18.3, TPI and RBCL) were up-regulated (UR) in EG after low temperature treatment (Figure 1A). Similarly, the enhanced abundance of these proteins that involved in the photosynthetic process has been reported under cold stress in Arabidopsis thaliana, winter wheat and rice [6]. The crucial roles of GAPA and GAPB played in the response of cold stress have been confirmed in previous researches [7]. As light 
receptors, LHCA4 and LHCB4.1 are closely associated with the capture and transmission of excitation energy [8]. Moreover, the function and significant role of TLP18.3 proteins for photosystem II repair has been verified in Arabidopsis thaliana. [9]. All these UR proteins work together to sustain the photosynthesis of plants during cold stress and play important roles in the cold tolerance of plants.

The result of Western blot was consistent with the proteomics analysis and showed that the expression of Rubisco large subunit (rbcL) was significant higher in EG, compared with CG (Figure 1B). The expression of rbcL induced by low temperature is directly linked with photosynthetic metabolism and plays a key role in the growth of plants. Thus, the low level of rbcL in CG may cause the significant reduction of carbon assimilation in plants and subsequently inhibit the photosynthesis and growth of plants. These results revealed that the cold stress seriously affected the photosynthetic process of plants and the influence degree was correlated with the cold tolerance of plant. The enhanced abundance of photosynthesis related proteins, especially the rbcL, can be considered as useful strategies to sustain the photosynthesis rate during cold stress.

Chlorophyll fluorescence can reflect the photosynthetic activities and the functional state of the photosynthetic system of plants [10]. As representative parameters of Chlorophyll fluorescence, maximum quantum yield of PSII (Fv/Fm), actual quantum yield of PSII (Y(II)), photochemical quenching (qP) and non-photochemical quenching (NPQ) have been widely used in the investigation of PSII activities of plants [11]. In our study, the relatively low values of Fv/Fm and Y(II) in CG reflected that the electron transfer at acceptor side of PSII was blocked under cold stress and suggested that the cold-induced damage of photosynthetic apparatus was more severe in CG leaves (Figure 2B) [12]. These results are similarly to previous studies previous studies performed in cucumber [13]. Moreover, the decrease of NPQ accompanied by an increase of qP in EG agree with previous studies, and could be an effective strategy to improve the photochemical efficiency and protect plants from photooxidation under cold stress conditions [14]. Our results show that the photosynthetic activity of plants was severely affected by the cold stress and the cold-induced protection mechanisms were closely associated with the cold resistance.

Electron transport can regulate the regeneration of RuBP which is the mainly limitation of photosynthesis. To survive the low temperature, many alpine plants have evolved the adaptive mechanism which can prevent the cold-caused damage of PSII by maintaining the stability of electron transfer system under the chilling temperature. Moreover, to sustain the photosynthesis of plants and protect plants from photoinhibition, proteins involved in electron transfer showed significant changes during the cold stress [6]. In present work, our results revealed that cold stress may directly inhibit the photosynthetic electron transfer of plants and lead to a significant decrease in electron transport rate (ETR) accompanied with an increase in excitation pressure (1-qP) (Figure 3A). Furthermore, the high level of the 1-qP will result in the accumulation of reactive oxygen species (ROS) [11]. On the other hand, the expression of PetE was also up-regulated in EG after low temperature treatment, indicating that low temperature stress can also impact the abundance of proteins which played a key part in electron transport chain of photosynthesis. These finding suggested that plants can cope with cold stress by adjusting photosynthetic electron transport system which play a crucial role in the cold-resistance of plants.

Cold stress can not only lead the depression of photosynthesis but also induce the accumulation of ROS which may lead to irreversible damages of membrane and photosynthesis system at cellular level. In the defence mechanisms against cold stress of plant, the antioxidant system has played a crucial role. Several studies have shown that low temperature stress can significantly up-regulate the 
expression of ROS scavenging enzymes such as superoxide dismutase (SOD), ascorbate peroxidase (APX), catalase (CAT), and glutathione peroxidase (GPX) [15]. As widely distributed enzymes in the plant cells, GPXs can eliminate $\mathrm{H}_{2} \mathrm{O}_{2}$ and lipid hydroperoxides by using GSH [16]. Moreover, APX could sustain the redox homeostasis by reducing the content of $\mathrm{H}_{2} \mathrm{O}_{2}$ through the ascorbateglutathione (ASC-GSH) cycle [17]. Higher GPX and APX activity can also protect and stimulate the growth of plants under chilling stress [18]. In our study, the relative activities and the abundances of GPX and APX showed remarkable increase in EG than in CG (Figure 4), suggesting that these proteins may represent the important cold-responsive proteins and are crucial for increasing cold stress tolerance of plants. Comparable results were also found in winter wheat [19]. Our results supported the notion that GPX and APXs, as the key ROS scavenging enzymes, were involved in the coordinated regulation of the homeostasis maintenance under cold stress and played significant roles in the improvement of cold resistance. The accumulation of ROS scavenging enzymes is frequently associated with the development of freezing tolerance in plants.

\section{Materials and Methods}

\subsection{Plant materials and treatment}

The martials we used were grown in different environment and showed significant discrepancy in the cold tolerance. Wild type (cold-tolerance) and domesticated (cold-sensitive) Rhododendron chrysanthum Pall. tissue seedlings were used as the experimental group (EG) and the control group (CG), respectively. Then, moved them into growth chambers, $4^{\circ} \mathrm{C}$ for $12 \mathrm{~h}$. The mixed sampling strategy has been adopted to eliminate differences between individuals and three biological replicates (i.e. six samples) were conducted in the present study.

\subsection{Chlorophyll Fluorescence Measurement}

Chlorophyll fluorescence of EG and CG leaves were carried out with the Maxi-version of the Imaging-PAM (Heinz Walz, Germany) with an integrated CCD camera enables view and records highly resolved digital images of the emitted fluorescence. The Imaging WIN version 2.39 software (Heinz Walz, Germany) was used for further analysis. All plants were dark-adapted for 20 min to measure the chlorophyll fluorescence (Fo, Fm, Y(II), NPQ and qP), and a detached leaf with was clamped onto the holder. Fv/Fm and electron transport rate (ETR) was recorded during a saturating photon pulse $\left(4,000 \mu \mathrm{mol} \cdot \mathrm{m}^{-2} \cdot \mathrm{s}^{-1}\right)$.

\subsection{Protein extraction}

Plant materials were ground into liquid nitrogen and mixed with lysis buffer ( $8 \mathrm{M}$ urea, $2 \mathrm{mM}$ EDTA, $10 \mathrm{mM}$ DTT and 1\% Protease Inhibitor Cocktail). The mixture was sonicated three times on ice using a high intensity ultrasonic processor (Scientz). The remaining debris were removed by centrifugation at $20,000 \times \mathrm{g}$ at $4^{\circ} \mathrm{C}$ for $10 \mathrm{~min}$. The protein in the supernatant was precipitated with cold $15 \%$ TCA at $-20^{\circ} \mathrm{C}$ for $4 \mathrm{~h}$. After centrifugation at $4^{\circ} \mathrm{C}$ for $3 \mathrm{~min}$, the remaining precipitates were washed with cold acetone three times. Finally, the protein was redissolved in the buffer $(8 \mathrm{M}$ urea, $100 \mathrm{mM}$ TEAB, pH 8.0), and the protein concentration in the supernatant was estimated with a 2-D Quant kit, according to the manufacturer's instructions.

\subsection{Proteomics and Bioinformatics analysis}

After extraction, proteins were digested into peptides. TMT labeling, HPLC fractionation and LC- MS/MS were then used to analyze and quantify the dynamic changes of the proteome. To ensure adequate coverage, three biological replicates (i.e. six samples) were collected. The MS/MS data were 
processed using the Mascot search engine (v.2.3.0). Tandem mass spectra were searched against the SwissProt Green Plant database. For protein quantification, the MASCOT software package in NCBI were used in present work. The Gene Ontology (GO) annotation proteome was derived from the UniProt-GOA database, and the proteins were classified by Gene Ontology annotation. Kyoto Encyclopedia of Genes and Genomes (KEGG) database was used to annotate protein pathway. The protein-protein interaction network was obtained from the String database and the interactions between proteins were performed using Cytoscape software (3.4.0).

\subsection{Western Blotting for Rubisco Large Subunit}

Proteins were extracted as the method described above, and $20 \mu \mathrm{g}$ of proteins were collected for Western blot analysis by using the Amersham WB system (GE Healthcare Life Science, USA). Rabbit anti-RbcL (Agrisera Vannas, Sweden) (1:1000 dilution) and Rabbit anti-GAPDH (Abways, China) (1:5 000 dilution) were used as primary antibodies. Amersham WB Goat anti-rabbit Cyth5 (GE Healthcare, USA) (1:1000 dilution) was used as the secondary antibody.

\subsection{Assay of Enzyme Activities}

$200 \mathrm{mg}$ of leaves with three biological replicates were used for the determination of enzyme activities and handled in accordance with the method of corresponding kit. Ascorbate peroxidases (APXs, EC 1.11.1.1) activities were detected according to the method of Mittova et al. [20]. Glutathione peroxidase (GPX, EC 1.11.1.9) activities were assayed as described by Drotar et al. [21].

\subsection{Statistical analysis}

All data are represented as means \pm SD with three biological independent replications in the present study. Statistical analysis was performed by using SAS 9.4. A value of $\mathrm{P}<0.05$ was considered a statistically significant difference.

\section{Conclusions}

Our study demonstrated that cold stress could directly inhibit the expression of photosynthesis related proteins, photochemical activity and photosynthetic electron transport, thereby decreasing the photosynthetic performance. This finding may shed light on the function of photosynthetic capacity in improving cold resistance of plants and indicate the complex connections exist between photosynthetic performance and cold tolerance of plants. Moreover, the imbalanced absorption and utilization of light induced by cold stress will lead to a severe over-accumulation of ROS, which will damage the photosynthetic apparatus of plant and lead up to a vicious circle. However, cold-tolerant plants can cope with the ROS-mediated damage more efficiently compared with sensitive ones by increasing the content and activity of ROS scavenging enzymes. Our results also reinforce the notion that manipulating the expression of antioxidant enzymes and improving the capacity of photosynthesis may be an effective strategy for elevating the cold resistance of plants. All these changes which can help plants to survive in low temperature are considered as the crucial parts of cold tolerance mechanisms and our results provide precious information on the molecular mechanism involved in cold tolerance.

Acknowledgments: This work was mainly supported by the National Natural Science Foundation of China (31070224) and the Science and Technology Department of Jilin Province (20130206059NY).

Author Contributions: XZ, SC and HX designed the research; SC and HW prepared the plant materials for sequencing. SC carried out bioinformatics analysis of data; $\mathrm{SC}$ and $\mathrm{HW}$ performed the experiments and statistical analyses; SC interpreted the data and wrote the manuscript. All authors read and approved the final manuscript.

Conflicts of Interest: The authors declare no conflict of interest. 


\section{References}

1. An, F.; Li, G.; Li, Q.X.; Li, K.; Carvalho, L.J.; Ou, W.; Chen, S. The Comparatively Proteomic Analysis in Response to Cold Stress in Cassava Plantlets. Plant Mol Biol Report 2016, 34 (6), 1095-1110, DOI: 10.1007/s11105-016-0987-x.

2. Hajek, J.; Bartak, M.; Hazdrova, J.; Forbelska, M. Sensitivity of photosynthetic processes to freezing temperature in extremophilic lichens evaluated by linear cooling and chlorophyll fluorescence. Cryobiology 2016, 73 (3), 329-334, DOI: 10.1016/j.cryobiol.2016.10.002.

3. Bresson, J.; Vasseur, F.; Dauzat, M.; Koch, G.; Granier, C.; Vile, D. Quantifying spatial heterogeneity of chlorophyll fluorescence during plant growth and in response to water stress. Plant Methods 2015, 11, 23, DOI: 10.1186/s13007-015-0067-5.

4. Su, L.; Dai, Z.; Li, S.; Xin, H. A novel system for evaluating drought-cold tolerance of grapevines using chlorophyll fluorescence. BMC Plant Biol. 2015, 15, 82, DOI: 10.1186/s12870-015-0459-8.

5. Zhou, R.; Yu, X.; Ottosen, C.O.; Rosenqvist, E.; Zhao, L.; Wang, Y.; Yu, W.; Zhao, T.; Wu, Z. Drought stress had a predominant effect over heat stress on three tomato cultivars subjected to combined stress. BMC Plant Biol. 2017, 17 (1), 24, DOI: 10.1186/s12870-017-0974-x.

6. Janmohammadi, M.; Zolla, L.; Rinalducci, S. Low temperature tolerance in plants: Changes at the protein level. Phytochemistry 2015, 117, 76-89, DOI: 10.1016/j.phytochem.2015.06.003.

7. Goulas, E.; Schubert, M.; Kieselbach, T.; Kleczkowski, L.A.; Gardestrom, P.; Schroder, W.; Hurry, V. The chloroplast lumen and stromal proteomes of Arabidopsis thaliana show differential sensitivity to short- and long-term exposure to low temperature. Plant J. 2006, 47 (5), 720-34, DOI: 10.1111/j.1365-313X.2006.02821.x.

8. Wientjes, E.; van Stokkum, I.H.; van Amerongen, H.; Croce, R. The role of the individual Lhcas in photosystem I excitation energy trapping. Biophys J. 2011, 101 (3), 745-54, DOI: 10.1016/j.bpj.2011.06.045.

9. Sirpio, S.; Allahverdiyeva, Y.; Suorsa, M.; Paakkarinen, V.; Vainonen, J.; Battchikova, N.; Aro, E.M. TLP18.3, a novel thylakoid lumen protein regulating photosystem II repair cycle. Biochem J. 2007, 406 (3), 415-25, DOI: 10.1042/BJ20070460.

10. Gao, M.; Qi, Y.; Song, W.; Xu, H. Effects of di-n-butyl phthalate and di (2-ethylhexyl) phthalate on the growth, photosynthesis, and chlorophyll fluorescence of wheat seedlings. Chemosphere 2016, 151, 76-83, DOI: 10.1016/j.chemosphere.2016.02.061.

11. Liu, M.; Zhang, Z.; Gao, H.; Yang, C.; Fan, X.; Cheng, D. Effect of leaf dehydration duration and dehydration degree on PSII photochemical activity of papaya leaves. Plant Physiol Biochem. 2014, 82, 85-8, DOI: 10.1016/j.plaphy.2014.05.003.

12. Shu, S.; Tang, Y.; Yuan, Y.; Sun, J.; Zhong, M.; Guo, S. The role of 24-epibrassinolide in the regulation of photosynthetic characteristics and nitrogen metabolism of tomato seedlings under a combined low temperature and weak light stress. Plant Physiol Biochem. 2016, 107, 344-53, DOI: 10.1016/j.plaphy.2016.06.021.

13. Jin, L.; Che, X.; Zhang, Z.; Li, Y.; Gao, H.; Zhao, S. The mechanisms by which phenanthrene affects the photosynthetic apparatus of cucumber leaves. Chemosphere 2017, 168, 1498-1505, DOI: 10.1016/j.chemosphere.2016.12.002.

14. Gururani, M.A.; Venkatesh, J.; Ganesan, M.; Strasser, R.J.; Han, Y.; Kim, J.I.; Lee, H.Y.; Song, P.S. In Vivo Assessment of Cold Tolerance through Chlorophyll-a Fluorescence in Transgenic Zoysiagrass Expressing Mutant Phytochrome A. PLoS One 2015, 10 (5), e012720, DOI: 10.1371/journal.pone.0127200.

15. Kasote, D.M.; Katyare, S.S.; Hegde, M.V.; Bae, H. Significance of antioxidant potential of plants and its relevance to therapeutic applications. Int J Biol Sci. 2015, 11 (8), 982-91, DOI: 10.7150/ijbs.12096.

16. Navrot, N.; Collin, V.; Gualberto, J.; Gelhaye, E.; Hirasawa, M.; Rey, P.; Knaff, D.B.; Issakidis, E.; Jacquot, J.P.; Rouhier, N. Plant glutathione peroxidases are functional peroxiredoxins distributed in several subcellular compartments and regulated during biotic and abiotic stresses. Plant Physiol. 2006, 142 (4), 136479, DOI: 10.1104/pp.106.089458.

17. Xu, J.; Yang, J.; Duan, X.; Jiang, Y.; Zhang, P. Increased expression of native cytosolic Cu/Zn superoxide dismutase and ascorbate peroxidase improves tolerance to oxidative and chilling stresses in cassava (Manihot esculenta Crantz). BMC Plant Biol. 2014, 14, 208, DOI: 10.1186/s12870-014-0208-4.

18. Zhang, W.; Zhang, H.; Ning, L.; Li, B.; Bao, M. Quantitative Proteomic Analysis Provides Novel Insights into Cold Stress Responses in Petunia Seedlings. Front Plant Sci. 2016, 7, 136, DOI: 10.3389/fpls.2016.00136. 
19. Xu, J.; Li, Y.; Sun, J.; Du, L.; Zhang, Y.; Yu, Q.; Liu, X. Comparative physiological and proteomic response to abrupt low temperature stress between two winter wheat cultivars differing in low temperature tolerance. Plant Biol (Stuttg). 2013, 15 (2), 292-303, DOI: 10.1111/j.1438-8677.2012.00639.x.

20. Mittova, V.; Volokita, M.; Guy, M.; Tal, M. Activities of SOD and the ascorbate-glutathione cycle enzymes in subcellular compartments in leaves and roots of the cultivated tomato and its wild salt-tolerant relative Lycopersicon pennellii. Physiol Plant. 2000, 110, 42-51, DOI: 10.1034/j.1399-3054.2000.110106.

21. Drotar, A.; Phelps, P.; Fall, R. Evidence for glutathione peroxidase activities in cultured plant cells. Plant Sci. 1985, 42, 35-40, DOI: 10.1016/0168-9452(85)90025-1.

(c) 2017 by the authors. Licensee Preprints, Basel, Switzerland. This article is an open access article distributed under the terms and conditions of the Creative Commons by Attribution (CC-BY) license (http://creativecommons.org/licenses/by/4.0/). 